\title{
La legitimización de la semiología psiquiátrica y su conjunción con los conceptos de "normal" y "patológico": una visión en la Era de la revolución (siglos XVIII-XIX).
}

The legitimization of psychiatric semeiology and its conjunction with the concepts of "normal" and "pathological": a vision during the Age of Revolution (18th-19th centuries).

\section{Diego Enrique Londoño ${ }^{\text {a }}$}

${ }^{a}$ Doctor en psicología. Psicoterapeuta, consulta externa en la Clínica la Inmaculada. Bogotá, Colombia.

Correspondencia: Diego Enrique Londoño (delp81@yahoo.com)

Recibido: 23/06/2013; aceptado con modificaciones: 08/11/2013

RESUMEN: Este artículo tratará una pregunta esencial: ¿Qué es lo que le va dar validez y legitimidad dentro del ámbito psiquiátrico a la semiología? Para responder, inicialmente se analizan las características, las reglas y algunas de las problemáticas para la configuración de signos como elementos semiológicos y clínicos; enseguida se da una mirada a lo que se entiende por normalidad desde la perspectiva de Georges Canguilehm. Se examinará entonces la historia y los orígenes filosóficos y médico-psiquiátricos de la semiología en lo que se llamó la Era de la revolución. Se sale de la esfera psiquiátrica para tocar el ámbito de la retórica y la oratoria, y su función normalizadora y categorizadora de ciertos signos clínicos relativos al lenguaje, para regresar a la psiquiatría y analizar cómo Michel Foucault de una manera original concibió la sintomatología y la semiología a partir del concepto de "instinto", el eje voluntarioinvoluntario, y la influencia de la neurología en la mitad del siglo XIX. Finalmente, se ve como la estadística y la eugenesia jugarán un papel igualmente importante en la conceptualización de la norma y por ende del discurso científico detrás de los signos clínicos establecidos.

PALABRAS CLAVE: semiología, epistemología, normalidad, facultad, aspectos históricos, filosofía, retórica, instinto, estadística.
ABSTRACT: This paper will address an essential question: What is it that will give validity and legitimacy to the psychiatric semeiology? To answer this, initially we analyze the characteristics, rules and some of the issues for configuring semiological signs and clinical elements; next we take a look to what is understood by "normality" from the perspective of Georges Canguilehm. We then examine the history, and the philosophical and medicalpsychiatric origins of semeiology during what is called the Age of Revolution. To continue, we leave the psychiatric field in order to take a glance of the field of rhetoric and oratory, and the categorizing and normalizing function of certain clinical signs related to language; then we come back to psychiatry and discuss how Michel Foucault conceived in an original way symptomatology and semeiology as a result of the concept of "instinct", the voluntary-involuntary axis and the influence of neurology in the mid 19 th century. Finally, we analyze how the statistics and the eugenics played also an important role in the conceptualization of the norm and therefore the scientific discourse behind the established clinical signs.

KEY WORDS: signs and symptoms, knowledge, normality, faculty, historical aspects, philosophy, rhetoric, instinct, statistics. 
ORIGINALES Y REVISIONES

\section{Introducción}

"Existe una continuidad entre el comportamiento humano juzgado normal y el que se tiene por patológico. Entonces, ¿cómo y dónde fijar el paso de lo normal a lo patológico? ¿Quién debe legítimamente hacerlo: el sujeto que sufre, el médico con el que se reúne, o la sociedad? El sujeto que sufre es a menudo poco consciente de su estado (delirio). El médico (y cada vez más el paciente) es el objeto de presiones diversas que apuntan a reconocer de la patología, allí donde quizás ella no existe, aquello que contribuya a aumentar formidablemente el consumo médico. La sociedad, es decir lo cultural, es dependiente del lugar y del momento, por consiguiente eminentemente variable en sus intereses (1)." Con esta cita del psiquiatra francés, Edouard Zarifian, arrancamos con algunos de los interrogantes que se van a desarrollar a lo largo de estos textos y que conciernen directamente la psiquiatría y la creación de su semiología. Una de las más importantes autoridades en epistemología e historia de la psiquiatría, el filósofo y psiquiatra, Georges Lantéri-Laura, cita en una de sus obras más importantes que "el conocimiento semiótico en psiquiatría, como en el resto de la medicina, constituye el origen de la psiquiatría-ya que sin la clínica no se podría localizar ni síndrome, ni enfermedad, ni estructura y se ignorarían incluso las cuestiones arregladas en otras esferas-y sin embargo éste permanece desprovisto de autonomía. Éste se funda sobre su propia historia y sus propias observaciones, pero hemos aprendido que aquel no observaba nada sin remitir en múltiples ocasiones a la antropología, a la psicopatología y a la patología misma (2)." Ahora bien, para entender mejor las cosas, a partir de Littré, el "síntoma" está en relación con el sentido, especialmente con la aparición del psicoanálisis el "síntoma" tendrá un significado y una relación con la subjetividad, mientras que el "signo" es un indicio o señal, y está en relación con el juicio de valor. La idea del médico es partir de un síntoma, observable por una gran cantidad de personas - lo que cae bajo el peso del sentido-, para transformarlo en signo, quien sería el producto de una combinación y de un trabajo crítico para no tener en cuenta sino los rasgos únicos dentro de una entidad nosológica. Existen cuatro tipos de signos: los propios - exclusivos a un diagnóstico-, los comunes con otras áreas de la medicina, los patognomónicos - donde un solo signo es suficiente para el diagnostico pero éste no es idéntico al síndrome o enfermedad-y los signos cardinales - donde un grupo de signos es necesario (al menos uno propio) para el diagnostico. De todas maneras es de resaltar que la conformación y la aparición de estos signos se han incrementado de manera exagerada con el tiempo y con la evolución de la disciplina. ¿Pero a qué se debe ese crecimiento en los signos y los diagnósticos psiquiátricos? Si se tiene en cuenta que la particularidad de la psiquiatría es la ausencia de marcadores biológicos - a penas se descubre un marcador biológico la enfermedad deja de pertenecer a la psiquiatría para pertenecer a 
ORIGINALES Y REVISIONES

otra especialidad de la medicina, como el caso de la sífilis patética que pertenece ahora a la neurología y a la medicina general-, ¿a qué se debe entonces este incremento desmesurado en menos de un siglo de la cantidad de signos, de trastornos y enfermedades mentales? Por el momento la respuesta es difícil de acordar, y para tratar de responder de alguna manera, aunque jamás exhaustiva, vamos a tratar de entender otro aspecto importante que permitirá al lector llegar a sus propias conclusiones: ¿Qué es lo que le va a permitir a la psiquiatría decidir qué es un signo normal o patológico? ¿Qué es lo que le va dar una validez y una legitimidad dentro del ámbito psiquiátrico a la semiología? Para esto daremos una mirada a la época de nacimiento del alienismo y de la psiquiatría, que concuerda justamente con el periodo histórico denominado la "Era de la revolución".

\section{¿Cómo se constituye un signo?}

"Debemos fijar la más gran importancia en los elementos extrínsecos y en las cualidades del contacto no verbal y verbal; pero solamente existe la semiología psiquiátrica si se extraen unas invariantes, idénticas, cualesquiera que sean las condiciones del contexto y las singularidades del contacto. Es decir que no sabríamos hablar de signos en psiquiatría si algunos elemento idénticos no aparecieran en la entrevista, ya sea que se trate de un sujeto que viene por su propia voluntad a consulta por trastornos que nos confía en una situación de contacto excelente, o ya sea en un peritaje judicial mal aceptado, donde el detenido hostil emplea digresiones y evasivas. La semiología psiquiátrica no puede realizarse como tal si no dispone de signos que se encuentren idénticos a pesar de las variaciones del contexto donde aparecen y las particularidades del contacto establecido (2)."

Georges Lantéri-Laura retoma el caso de las obsesiones como signo clínico e imparte dos condiciones que hacen de las obsesiones, en ejemplo, signos legítimos de la clínica psiquiátrica: primero porque anteriormente muchos alienistas y clínicos las habían caracterizado, identificado y descrito en sus variedades, introduciéndolas en un thesaurus semioticus; y segundo, las obsesiones son signos clínicos porque se trata de aspectos diferentes de la norma corriente. LantériLaura propone tres puntos de referencia para aceptar a las obsesiones (como ejemplo) como signos clínicos. Primero, éstas hacen sufrir al paciente en múltiples ocasiones, de manera que no se trata de una singularidad fugaz sino de una iteración de la misma molestia típica. Segundo, las obsesiones se encuentran de un paciente al otro, si no son exactamente las mismas por lo menos sí se mantienen los mismos rasgos formales que permiten reconocerlas fácilmente. Y tercero, 
las obsesiones deben distinguirse de otras alteraciones igualmente típicas de la experiencia interior, por ejemplo una obsesión es obsesión porque no es una fobia o una fuga de ideas (2). El signo adquiere su poder designativo, por el cual el concepto es juntado en un enlace necesario al significante, gracias a su oposición a los otros signos. Para Lantéri-Laura las obsesiones no son un elemento aislado en la semiología psiquiátrica sino que tienen un lugar propio y diferenciado dentro de un grupo bastante homogéneo de afecciones de la experiencia vivida (2). Como podemos ver, Lantéri-Laura enuncia una aparente lógica formal para la conformación de signos clínicos. Empero, él es el primero en cuestionar esta misma lógica haciendo entender que ciertos elementos no merecen el calificativo de signos clínicos a pesar de ser reconocidos por muchos clínicos como tales, es el caso del suicidio o de las tentativas de suicidio, o de los trastornos de la conducta. Para el maestro en psiquiatría, el suicidio no es un signo psiquiátrico porque muchos suicidios están en relación con cuestiones personales y valores sociales que no tienen nada que ver con la patología mental, y segundo, se trata de un conjunto bastante complejo que no se deja tratar como una unidad semiótica. Estos elementos son demasiado complejos, demasiado globales y demasiado implicados en múltiples redes como para poder ser considerados como unidades semióticas. Sin embargo, el suicidio o la tentativa de suicidio pueden aparecer o ser determinados por una afección mental, por ejemplo del desencadenamiento psicótico, o puede ser la etapa por la cual se concretiza una delirio altruista en la melancolía. De todos modos, y como buen estructuralista, Lantéri-Laura afirma en otro texto (3) que una conducta no es suficiente para determinar un diagnostico. Por otro lado, el psicoanalista Pierre-Henri Castel aduce que si "tal signo X de la locura no tiene una significación social eso no quiere decir que ese $\mathrm{X}$ sea directamente biológico, pero tampoco quiere decir que si ese $\mathrm{X}$ no es biológico, y está desprovisto de una significación social, que X no sea real. Es difícil tratar esos signos o la locura como una realidad natural sin dar testimonio al mismo tiempo del tipo de cultura y su concepción de la moral o el sufrimiento psíquico que nos impregna (4)". Por consiguiente se puede esperar que la psiquiatría pueda determinar qué es o no es un signo patológico según un cuadro y un marco social en el cual ella está inscrita.

Signo normal y signo patológico: la "ciencia" detrás de todo esto

La problemática de lo normal y lo patológico, de lo normal y lo anormal en medicina ha sido ampliamente discutida por muchos teóricos e historiadores, sin embargo es tal vez la obra del médico Georges Canguilhem (5) quien se presente 
ORIGINALES Y REVISIONES

como una de las más completas en relación a estos conceptos. Para él, la salud es la capacidad de gozar con las normas existentes y resulta de esas normas existentes del organismo; la enfermedad pone al organismo a inventar nuevas formas de funcionamiento, nuevas normas. "Una norma se propone como un modo posible de unificación de lo diverso, de resorción de una diferencia, de arreglo de un diferendo. Pero proponerse no es imponerse. A diferencia de una ley de la naturaleza, una norma no necesita su efecto. Es decir que una norma no tiene ningún sentido de norma totalmente sola y totalmente simple. La posibilidad de referencia y de arreglo que ella ofrece contiene, del hecho de que no se trata de una posibilidad, la latitud de otra posibilidad que sólo puede ser inversa. Una norma, en efecto, es sólo la posibilidad de una referencia cuando ha sido instituida o escogida como una expresión de preferencia y como instrumento de una voluntad de substitución de un estado de cosas satisfacientes por un estado de cosas decepcionantes (5)." A pesar de lo que la fisiología significa para la medicina y la biología humana, el concepto de norma no se deja reducir a un concepto objetivo determinado por los métodos científicos. En muchas ocasiones la norma es una cuestión de acuerdos y de negociaciones, es una cuestión que escapa al ámbito biológico o puramente médico o estadístico, y salta al campo social y antropológico, e incluso subjetivo, quienes terminan por indicar esas preferencias de las que habla Canguilhem (5). Como lo afirma él mismo, no existe una ciencia biológica de lo normal, existe una ciencia de las condiciones y las situaciones biológicas normales la cual traduce la relación de las ciencias de la vida a la actividad normativa de la vida y a las técnicas biológicas de producción y de instauración de lo normal (5). Esta ciencia es la fisiología. Pero, ¿y cómo aplicar esto al campo de la psicopatología? ¿Las normas biológicas y somáticas se aplican por igual al ámbito mental y psicológico? Para algunos autores, como Castel (4), la psiquiatría y la psicopatología (sin importar su orientación: psicoanalítica, neurocientífica, conductista, etc.) sí pueden determinar una norma objetiva de la locura porque éstas no tienen ningún ideal preconcebido de la normalidad que alcanzar, ellas tienen que negociar y debatir entre las razones en favor de diagnosticar según ciertos parámetros la locura o la enfermedad mental (como el suicidio), con las razones o los argumentos que se erigen en contra de estos parámetros (4). Y devolver a la norma a un organismo o a un ser que se alejaron de ésta, es la función de sanación del galeno. Para el psiquiatra es cuestión de traer a la norma al sujeto que se alejó de la representación normal común en un momento dado y un contexto preciso de lo que es la salud, o en su defecto, la cordura. De ahí que alguien como Lantéri-Laura utilice la noción de "norma corriente" como medida para dictaminar como patológicos los signos que se alejen de ésta misma. No obstante, algunos autores van a dar su perspectiva del asunto en lo que atañe a la psicopatología y a la normalidad de la mente. Para Freud (6) la normalidad es una "ficción ideal", y entre la neurosis y 
la normalidad existirían "transiciones graduales"; para el padre del psicoanálisis entre la neurosis (término redefinido por Freud y Janet) y la normalidad existiría una continuidad y ambas adquirirían tintes de la una y de la otra. Se trata de un valor convencional acordado el cual establece los límites entre lo normal y lo anormal, sin ser necesariamente lo anormal algo patológico.

Las sensaciones y la razón en el periodo pre-pineliano:

La cuestión de las facultades

Desde los griegos hasta el siglo XVIII, esencialmente, la locura se dividía simplemente en manía y melancolía. Para el médico savoyardo Joseph Daquin, quien publicó en 1791 su Ensayo sobre la filosofía de la locura (7), existen otros tipos de loco: el loco de atar, el loco tranquilo, los extravagantes, los insensatos, los imbéciles y los dementes. Lo que según Daquin - considerado por algunos como el verdadero padre del alienismo francés-es común a todas estas formas de locura, además de estar cada una escalonada a diferentes grados de enajenación, es el fallo de la razón. Ella misma sería la facultad principal de la mente humana, a diferencia del animal, la cual le permite conocer la verdad en tanto que ésta le es necesaria para su subsistencia y con la cual la obviedad de los objetos golpea a su mente. En cada una de las formas de la locura, la facultad intelectual se ve alterada o afectada de una manera u otra. Son los propósitos de cada uno de los locos, el carácter de los discursos, la forma y el tipo de las ideas que enuncian, la utilización del lenguaje, que sirven de guía semiótica; hay toda una seria de comportamientos típicos que se desdibujan y que la observación de Daquin utiliza para clasificar en las seis variedades de locura. Hay que partir de Francis Bacon y Descartes, quienes retomando a Platón y sus tres facultades del alma, proponen las tres facultades de la mente: la memoria, la imaginación y la razón. Para Daquin esta última es la corona del entendimiento, es el conocimiento de cómo debemos arreglar las operaciones del alma. Las sensaciones vienen enseguida a jugar un papel importante a la hora de excitar el cerebro, según W. Cullen, si la sensación sólo estimula una parte del cerebro entonces resultan falsas percepciones y falsas asociaciones.

Sin embargo para entender mejor el abordaje de Pinel y los primeros alienistas de los signos particulares que harán parte, en tanto patológicos, del diagnostico y la legitimación de éste, hay que comenzar a ver en la corriente sensualista iniciada por John Locke y Etienne Bonnot de Condillac las primeras aprehensiones de estos signos. Esta corriente filosófica planteaba la idea de que todo el conocimiento y todas las ideas provenían de los sentidos. Para Locke el 
ORIGINALES Y REVISIONES

conocimiento en el Hombre aparece mediante la confluencia de ideas simples e ideas complejas, las primeras son las ideas que no se nos pueden ser comunicadas o transmitidas, solamente se adquieren a través de la experiencia, como la sensación de calor, frío, etc., y es completamente imposible engendrar una idea simple nueva (8). Las ideas complejas son la combinación de las simples. Para Locke el conocimiento verdadero y objetivo es la percepción de una relación entre las ideas. Por otro lado, para Condillac toda sensación puede engendrar una facultad, y el empleo de signos para nombrarlas y hacer abstracción de ellas las extiende; el lenguaje es la esencia y la base de toda forma de análisis (8). Para este filósofo, los fenómenos brutos de un campo deben ser clasificados y nombrados, una observación puramente objetiva es de todos modos intuitiva, en consecuencia se deben crear conceptos que deberán "adquirir una estructura enunciable" (9). La psicología de las facultades, proveniente de la escuela escocesa del sentido común, ha abierto también un campo bastante amplio sobre los elementos a tener en cuenta dentro de la semiología psiquiátrica. Para esta escuela el "sentido común" designa las "leyes fundamentales" de la creencia; las reglas esenciales e inmediatas, necesarias y universales. Si para Condillac y Locke la percepción estaba separada del juicio, en tanto que el sentido era una simple facultad receptiva, para la escuela escocesa en la lengua común la palabra sentido implica necesariamente el juicio (buen sentido, sin sentido, sentir) (8). Thomas Reid fue el gran pensador de esa corriente quien propuso una serie de facultades psicológicas descriptivas y categorizadas, basadas en el juicio, para dar cuenta del conocimiento y de la razón. Reid habló de las facultades de la memoria, del aprendizaje, de la inteligencia, de la percepción y de la voluntad. Por consiguiente, las alteraciones del pensamiento, del humor y la vida afectiva, de la conciencia, de la percepción, del sentido crítico, etc., se han deducido poco a poco como variaciones mórbidas provenientes en gran medida de esas facultades psicológicas que se erigen como los elementos indispensables a la hora de capturar, nombrar, clasificar y configurar un signo.

\section{Lo que heredó Pinel}

Según ciertos autores, como Bercherie, Pinel le da un estatuto científico a la investigación y observación de la locura a partir de una mirada médica. El sería, siguiendo los preceptos de los Ideólogos — sobre todo en medicina-, el fundador de la psiquiatría como una idea de ciencia o pre-ciencia, como una ideología, en su sentido epistemológico (9). "Uno puede naturalmente interrogarse sobre esta epistemología un poco ingenua, pero un hecho se mantiene: Pinel abre allí la 
exploración sistemática de un campo y la organización de los fenómenos que lo constituyen. Este proceso ha sido el fundamento sobre el cual se constituyó enseguida un saber realmente positivo de la psiquiatría, una vez que, como a menudo en el nacimiento de las ciencias, un descubrimiento inesperado habrá proporcionado un método nuevo para explorar y clasificar los fenómenos (9)." Para tal fin los métodos de los naturalistas, como Carl von Linné o el conde de Buffon, van a permitir que prosiga el saber psiquiátrico de una manera organizada y metódica, suponiendo que toda las problemáticas anteriores han sido resueltas, la clasificación al mejor estilo de la botánica va a tomarse toda la medicina y va a permitir la creación de los primeros tratados taxonómicos en el siglo XVIII. Aunque ya se tenían registros de los primeros tratados taxonómicos desde el Imperio Romano, en especial el de Areteo de Capadocia (10), médico griego del siglo I d.C., donde la manía, la melancolía y la epilepsia ya tenían sus primeros signos clínicos y sus indicaciones terapéuticas. La cuestión de los humores era trascendental, la melancolía era una enfermedad aguda de la bilis negra, donde prevalecían algunos signos como la irascibilidad, la tristeza, o la sumersión en un abatimiento profundo. La visión de Pinel en cuanto a la manía y a la melancolía en las dos versiones de su Traité médico-philosophique sur l'aliénation mentale no están muy alejadas del abordaje particular semiológico de Areteo de Capadocia, son muy parecidos ambos en cómo cada uno retoma la cuestión a partir del concepto de delirio general y delirio parcial o exclusivo, o del tema del abatimiento profundo y la inclinación hacia la desesperanza.

Como bien lo resalta Lanteri-Laura (2) lo que hace que un signo como el abatimiento profundo o el "dolor moral" sean signos legítimos del diagnostico de la melancolía, es que ya en el pasado otros los hayan reconocido como tal, la semiología es antes que nada un saber cumulativo y heredado; y segundo, para este epistemólogo de la psiquiatría lo que hace que, por ejemplo, la fuga de ideas se constituya bien como un signo que se pueda aislar como tal, es el hecho de que ésta se presente correctamente como un signo para que se pueda enseguida establecer el diagnostico de manía.

La retórica y la semiología psiquiátrica: las pasiones internas y los signos externos

Uno de los elementos del saber que más ha influenciado la semiología psiquiátrica desde el momento en que apareció el alienismo a finales del siglo XVIII y principios del XIX fue tal vez la retórica. La gran tesis doctoral del profesor de literatura francesa de la Universidad de Fribourg (Suiza), Juan Rigoli (11), mues- 
ORIGINALES Y REVISIONES

tra con hincapié - como lo señalan Alvarez, Esteban y Sauvagnat (12)—cómo la mayor parte de los cuadros clínicos surgidos en el siglo XIX fueron descritos siguiendo los lineamientos y las convenciones propias a la retórica del siglo XVII al siglo XIX. Los signos retomados a la retórica mantienen una referencia a ella mediante la noción de un verosímil y un aceptable (12).

Personajes como Pinel, Esquirol o Leuret, fueron marcados en su formación juvenil por la filosofía y el arte de la retórica y las bellas-letras en especial por el tratado de Charles Rollin, De la manière d'enseigner et d'étudier les belleslettres, par rapport à l'esprit et au coeur, de 1726-1728, o incluso las lecciones de literatura antigua y moderna, el Gradus ad Parnassum y numerosos textos de literatura clásica como las Leçons de litterature et de moral de Noël y Deplace, entre muchísimos otros (11). Todo lo que concierne el lenguaje de las "pasiones" (del griego pathos, "sufrimiento", "enfermedad"), a las que Cicerón en sus Disputaciones Tusculanas definió como aquello que perturbaba o alteraba el ánimo, pusieron el acento sobre la elocuencia y los instintos del orador. Para Aristóteles en las artes de la persuasión retórica, el pathos era el medio por el cual se debía tocar la sensibilidad y la emoción del auditor, a diferencia del ethos el cual debía remitir su fuerza de persuasión a la integridad del orador. La pasión (pathos) es un elemento que va a ser de gran importancia en la retórica desde sus inicios y más tarde en la lingüística, pero también tocará al campo de la medicina y el alienismo. En la obra de Esquirol (13) y de Pinel el concepto de pasión está muy presente en el origen de la locura y de sus causas. Las pasiones van tanto a influenciar al arte de la retórica como éste último al alienismo. La semiología médica se manifestará como los signos exteriores de las pasiones internas del alma. De ahí que muchos tratados de semiología médica del siglo XVIII y XIX trataran los efectos exteriores de las pasiones del alma. La principal de estas manifestaciones, para un alienista como Guislain, son las expresiones del rostro las cuales delatarían las emociones y las pasiones que dominan al alienado (11). Pero para muchos otros alienistas es a través del lenguaje, la voz, el discurso y la escritura que se podrán de verdad desvelar estas pasiones mortíferas del alma. En el famoso Dictionnaire de sciences médicales par une société des médecins et de chirurgiens, en un artículo de 1821 (14) los autores retomando la noción de "signo" y sus "efectos", explican el paso que se va a realizar de la estética de la retórica y sus elementos principales (dispositio, elocutio, pronuntiatio) a la semiología médica. "Los 'efectos' del 'alma' sobre la palabra... se redoblan de un 'efecto' de la palabra sobre el 'alma' de aquel que la recibe. Las pasiones son a la vez sufridas y suscitadas; el discurso no es únicamente depositario sino generador de afectos; el 'hombre que manifiesta su pensamiento y el estado de su alma' no está reducido a una simple pasividad; si él está 'agitado por la pasión' éste agita también las pasiones del prójimo. Aún más: el efecto 'poderoso' que él produce se retorna, directa o indirectamente, contra él (11)." 
El uso de la palabra y la voz serán signos exteriores de la locura; la mirada del alienista sobre el lenguaje empleado en los escritos de los enajenados pasa por una evaluación de tipo retórico a un punto en el cual se superponen el léxico clínico y la tópica literaria. Como nos lo recuerda Rigoli, "es la expresividad emotiva del lenguaje, vinculada al mito del origen afectivo de las lenguas, y la retórica de las pasiones, quienes atraviesan y reorientan el discurso de los alienistas; una de las convicciones menos declaradas, pero sí de las más firmes del alienismo, es que "el lenguaje hablado tenga su fundamento natural en las expresiones sensitivas y de sentimientos'. Las representaciones médicas de la palabra exaltada del alienado, arrastrado por esta pasión exacerbada que es la locura, hacen curiosamente retomar la expresión del loco a ese punto de origen designado por Rousseau donde la pasión omnipotente precede y genera el lenguaje... (11)."

\section{Los anormales de la psiquiatría: Foucault y el "instinto"}

Los Anormales es el título del curso que dictó Foucault en el Collège de France entre 1974 y 1975 (15). Para este autor, a partir de un análisis de la cuestión médico-jurídica o médico-legal, y del involucramiento de la psiquiatría y el alienismo dentro de esta esfera, buena parte de la semiología y la sintomatología psiquiátrica nacen a partir de la problemática del concepto de "instinto". Según este filósofo, la psiquiatría conceptualiza el vocablo instinto a partir del tristemente célebre caso de Henriette Cornier quien en 1825 decapitó a una niña sin motivo alguno ${ }^{1}$. Justamente el hecho de que la homicida no presentara un estado de delirio contundente, no presentara alienación mental visible, todavía menos motivos del crimen atroz, llevó a la psiquiatría a una transformación de las llamadas locuras parciales, pero especialmente la llevó a postular al instinto como motor de la enajenación, así sea parcial, dentro de las operaciones discursivas de la misma. "Es a partir de la noción de instinto que toda la problemática de lo anormal podrá organizarse alrededor de lo que era antes el problema de la locura, de lo anormal a nivel de las conductas más elementales y más cotidianas (15)." Lo que según Foucault ha permitido que este concepto de instinto haya adqui-

\footnotetext{
La psiquiatría y el alienismo se interesan en los crímenes y en el peritaje porque según Foucault estas dos no se constituyen inicialmente como una especialidad de de la medicina sino que se consolidan como una de las ramas de lo que se conoció como la «higiene pública». Esta última nace de una preocupación por combatir las epidemias masivas (cólera y tuberculosis) que azotaron a Europa durante el siglo XIX. La psiquiatría viene como higiene y protección del cuerpo social contra los peligros de la locura, por consiguiente era necesario una doble codificación: la locura como una enfermedad, su patologización, crear toda una jerga médica alrededor de ésta para acercarla a la higiene pública; y la segunda codificación es la de asimilar la locura a la peligrosidad, la locura portadora de un cierto número de riesgos para el orden social.
} 
ORIGINALES Y REVISIONES

rido tanta fuerza dentro de toda la actividad psiquiátrica sin que siquiera ésta lo haya notado es gracias a tres factores fundamentales que conciernen la inserción de la psiquiatría en los mecanismos de poder (poder exterior a ella): en primer lugar se trata de una reforma administrativa, la ley de 1838, instancia que va a dar la legitimidad a la psiquiatría de internar sujetos y de recibirlos en asilos y hospitales; segundo, la reorganización de la demanda familiar, con la ley de 1838 la familia o el entorno pueden pedir que el familiar enfermo sea internado; y el tercer proceso que entra en juego es la aparición de una nueva demanda política a la atención de la psiquiatría. Esta demanda política consiste en pedirle a ésta que provea un discriminante psiquiátrico-político entre individuos o un discriminante psiquiátrico con efecto político entre individuos, entre grupos, entre ideologías, y entre los mismos procesos históricos.

Para Foucault, entre 1845 y 1850 aparece la verdadera psiquiatría, y desparece el alienismo, el de Esquirol y Pinel, donde la noción de la verdad era la que jugaba el papel más importante; a partir de 1845-1850 es la fusión de la psiquiatría con la neurología y la cuestión del doble juego de la acción voluntaria e involuntaria que se torna en algo apremiante; el loco es aquel donde la jerarquía entre lo voluntario y lo involuntario se encuentra perturbada; ni los delirios a grandes rasgos, ni el frenesí, ni la demencia son necesarios para esta nueva disciplina que es la psiquiatría. Ya no serán necesariamente las facultades mentales, principalmente la razón y la moral, quienes tengan que verse afectadas para dictaminar la enajenación o una forma de locura. Ya no importa la relación del sujeto con la aprehensión de la verdad, ahora son los ejercicios involuntarios de las facultades, lo instintivo y lo automático - la pérdida de uno como agente causal de sus actos-como nuevos ejes de constitución de la semiología psiquiátrica.

Si los primeros alienistas aprehendían una conducta o fenómeno como mórbido era porque éste guardaba algún fragmento de delirio en su interior, para los psiquiatras de mitad del siglo XIX, en especial a partir de Baillarger en Francia y Griesinger en Alemania, lo que hará de una conducta un signo clínico es, lo primero de todo, su distanciamiento o desviación de la norma, de las reglas de conformidad que fueron definidas desde un trasfondo administrativo, familiar y político que ya vimos más arriba; y segundo, una conducta es signo según como ésta se sitúe en el eje involuntario-voluntario. El distanciamiento de la norma y el hundimiento en el automatismo instintivo serán las dos referencias, según Foucault, que van a establecer la nueva semiología psiquiátrica y los comportamientos señalados como mórbidos. 
ORIGINALES Y REVISIONES

La estadística, la norma y el distanciamiento de la norma: Quetelet, el "hombre promedio", y la eugenesia de Galton

La eugenesia fue una doctrina social que se formó a finales del siglo XIX en los países mayoritariamente anglo-sajones y que promovía la idea, muy en boga en la época, de un mejoramiento de la herencia de todos los factores y rasgos biológicos, intelectuales y fisiológicos humanos. El británico Francis Galton fue el padre de la eugenesia, durante años sistematizó la idea de una "selección artificial" o "selección racional" al contrario de la "selección natural" de Darwin. A través de estudios biométricos y estadísticos, siendo él el fundador de lo que se conoce como la biometría o la bio-estadística, le dio forma a los elementos que conforman la estadística moderna para tratar de, a partir de las visiones de Darwin, poner en funcionamiento la posibilidad de un mejoramiento biológico de la especie humana.

A diferencia del astrónomo y matemático Alphonse Quetelet, Galton no buscaba el hombre común u hombre promedio (homme moyen), él buscaba las diferencias y las particularidades de cada individuo, sus aptitudes naturales. Mientras que Quetelet se interesaba en los hombres medios o promedios como un valor central en torno al cual una medida característica humana se agrupaba de acuerdo a una curva normal, Galton se interesaba en la distribución relativa de hombres no promedio, pero si creía, como Quetelet, que existían ciertos atributos humanos distribuidos en una curva alrededor de un valor normal (16). Para Galton lo que va a ser interesante de observar no es tanto que existan una norma o un hombre promedio en general, sino que existan individuos que se alejen de esa norma, hecho que él va llamar la ley de desviación, y que va a fundar la estadística moderna. Como lo define el sociólogo Alain Desrosières (16), Galton va a crear un espacio de medida para una serie de objetos o nociones que parecían hasta entonces inconmensurables: las aptitudes humanas (16). Quetelet intentó hacerlo con algo relativamente sencillo de aprehender y de transponer al mundo cifrado, la estatura, pero Galton va a ir más lejos y va a tratar de deducir a partir de las medidas estadísticas el origen de los genios o superdotados. Este autor se va a centrar en la ascendencia de personajes como Bach, Darwin o Bernoulli para probar la hipótesis de una herencia en línea directa de la genialidad. Para Galton, ni el entorno, ni la educación, ni la crianza, parecen tener mayor importancia. Por ejemplo, este autor utilizó una investigación sociológica sobre la pobreza en Londres y la posición de los hogares en una escala social y económica con ocho grados o valores, como medio para afianzar sus hipótesis. Esta investigación se realizó para contar y comparar las categorías de pobreza según los barrios de Londres, pero Galton se sirvió de ella como un indicador de aptitudes naturales, desde una perspectiva naturalista y esencialista, asimilando unos "valores cívi- 
cos" a "valores genéticos" naturales (16). De acuerdo con el padre de la eugenesia moderna, los valores cívicos ya estarían inscritos en el cuerpo y harían parte de su esencia, estos se transmiten de padre a hijo, y de generación en generación. Lo que es importante de remarcar es que Galton asimila lo que es codificado en esa investigación social como atributos biológicos comparables a la estatura o a la fisionomía. Si para Quetelet la estatura promedio, del hombre promedio, permitía colegir la existencia de un objeto más general, para Galton la distribución normal es supuesta, lo que se codifica en la investigación socio-económica lo toma como un atributo natural heredado que se puede medir (16).

Esto va a permitir a que más tarde las aptitudes (inteligencia, emociones, personalidad, etc.) puedan ser medidas y objetivadas, transformadas en cifras y en conceptos claves para la medición de su frecuencia y para establecer diagnósticos y leyes probabilísticas sobre la etiología o sobre los tratamientos, pero más importante en lo que nos concierne, para establecer signos particulares (e.g. el test de inteligencia de Binet y Simon, o los tests de personalidad, entre otros). Todos estos elementos que se reconceptualizan, generaran una medida: se puede suponer entonces que para hacer ciencia y tener algún tipo de medida infalible objetiva una serie de conceptos mentales abstractos deben reducirse de modo que puedan ser parametrables y por lo tanto se puedan dar el lujo de juzgar, de distinguir claramente entre el hombre "normal" y "anormal", el que se aleja de la norma y el que se le acerca. La eugenesia abre una puerta y genera una ruptura sin precedentes en los anales de la clínica, no tanto a partir de la noción o de la idea de que todas las aptitudes humanas o los tipos humanos (genio, criminal, retrasado, enfermo mental, etc.) sean heredados - idea que ya existía en la psiquiatría - sino en el hecho de que esos atributos puedan ser medidos y cifrados para tomar decisiones, legitimar teorías y semiologías clínicas.

\section{Conclusión}

Como se puede observar, este artículo no es exhaustivo, se quedan por fuera otras corrientes y disciplinas que igualmente aportaron al desarrollo de un thesaurus semioticus, como es el caso de la anatomopatología, el conductismo, el psicoanálisis, la fenomenología (como corriente filosófica) o las ciencias cognitivas, entre otras. Todas estas orientaciones suministran particularidades diversas y disimiles a este campo complejo, pero autorizan la distinción interna y el reconocimiento de las distintas aptitudes inherentes al sujeto de la psiquiatría. "De la caracterización de esas diferencias arranca la semiología cuyo desarrollo transita de lo uno a lo múltiple, de lo tosco a lo sutil, de los grandes fenómenos 
a los pequeños indicios, de lo genérico a lo elemental, tal como se puede observar de forma meridiana en los estudios sobre las alucinaciones que van desde Esquirol hasta Clérambault, pasando por Baillarger y Séglas (17)." Muchos de estos signos provenientes de estas orientaciones son en su fondo polisémicos y la operación de su combinación intenta buscar la monosemia, por consiguiente, el diagnóstico (18). Se remite al lector a la obra de Rafael Huertas (19) para una mirada más en profundidad del acontecer semiológico de la psiquiatría, y la conceptualización de lo normal y lo patológico durante el siglo XIX. De igual manera el artículo de Manuel Desviat (20) aporta una visión crítica del síntoma en la actualidad y el orden social en donde éste se asienta y adquiere sentido.

Por un lado vemos como la semiología es un sistema heredado y cumulativo, existe una transmisión de generación en generación de una cierta cantidad de signos considerados mórbidos y ya legitimados con anterioridad. Por el otro lado, la filosofía ha hecho su parte de contribución, a través de la percepción y las sensaciones, por un lado, y por el otro el de las facultades en tanto poder de acción o potencia, el cual "expresa una idea de la razón primera, cualquiera que ésta sea, la cual vuelve a un objeto capaz de llevar a cabo las acciones que éste último manifiesta (21)." Es a partir de estas facultades que, retomadas a las corrientes filosóficas ya mencionadas, debemos observar "el principio o el origen de los fenómenos que el (21)" objeto de la psiquiatría presenta. Estos fenómenos son una cuestión de arreglo y de debate al interior de la psiquiatría misma, la cual por estudiar y mantener una tradición epistemológica seria recibe una legitimidad a la hora de considerar nuevos signos o de tener que descartarlos, con la posibilidad de que ésta misma pueda estar sujeta a un debate interno donde se discutan los pros y los contras de una decisión consecuente.

La retórica y la oratoria determinaron igualmente las características de una "lengua del enajenado" o el objeto del alienismo naciente, y así, demarcaron esas variaciones que el alienista debía detectar en la lengua del loco, así como lo que de esta lengua se alejaba de la norma. Por el otro lado, los instintos (las pasiones) y los aspectos motores para Foucault trazaron la división entre los signos normales y patológicos mediante su fusión con la neurología y las cuestiones de los actos voluntarios e involuntarios. Esto cambió hasta cierto punto la dirección que esta semiología del alienismo tomaba con respecto a las facultades, encaminándola más hacia una inclusión de la neurología como nueva fuente de signos. Mientras que la estadística, la construcción de ésta misma y las escalas evaluativas, facilitaron no solamente el diagnóstico, también determinaron los elementos y los signos a tomar en cuenta a partir de una objetivación y una cosificación de las aptitudes humanas en un contexto socio-cultural proclive a esto; y al mismo tiempo sirvieron de discriminantes entre lo normal y lo patológico, lo normal y lo anormal. 
ORIGINALES Y REVISIONES

\section{BIBLIOGRAFÍA}

(1) Zarifian E. Des paradis plein la tête. Paris: Editions Odile Jacob, 1994.

(2) Lantéri-Laura G. Psychiatrie et connaissance. Paris: Science en situation, 1991.

(3) Lantéri-Laura G. Normal et pathologique: esquisse d'un point de vue médical. En: Bing F, Braunstein JF, Roudinesco E, editors. Actualité de Georges Canguilhem. Le normal et le pathologique. Paris: Les empêcheurs de penser en rond, 1998 ; p. 47-58.

(4) Castel PH. L'esprit malade. Cerveaux, folies, individus. Paris: Éditions Ithaque, 2009.

(5) Canguilhem G. Le normal et le pathologique. 11th. edition. Paris: Quadrige/Presse Universitaire de France, 2011.

(6) Freud S. Esquema del psicoanálisis y otros escritos en doctrina psicoanalítica. Madrid: Alianza editorial, 2009.

(7) Daquin J. La philosophie de la folie ou Essai philosophique sur le traitement de personnes attaquées de folies. Paris: Née de la Rochelle, 1792.

(8) Bréhier E. Histoire de la philosophie. 5th. Edition. Paris : Quadrige/PUF, 2004.

(9) Bercherie P. Les fondements de la clinique. Histoire et structure du savoir psychiatrique. Paris : La Bibliothèque d'Ornicar?/Seuil, 1980.

(10) Areteo de Capadocia (Aretée de Cappadoce). Traité des signes, des causes et de la cure des maladies aiguës et chroniques. Paris: Editions Lagny, 1834.

(11) Rigoli J. Lire le délire. Aliénisme, rhétorique et littérature en France au XIXe. siècle. Paris: Fayard, 2001.

(12) Álvarez JM, Esteban R, Sauvagnat F. Fundamentos de psicopatología psicoanalítica. Madrid: Síntesis, 2004.

(13) Esquirol JE. Des passions considérées comme cause, symptômes et moyens curatifs de l'aliénation mentale [tesis de medicina]. Paris: Ecole de Médecine de Paris, 1805.

(14) Fodéré FE. Signe. En: Dictionnaire des sciences médicales-par une société des médecins et de chirurgiens. Vol. 51. Paris: C.L.F. Panckoucke, 1821; p. 267-306.

(15) Foucault M. Les Anormaux. Cours au Collège de France, 1974-1975. Paris: Seuil/Gallimard, 1999.

(16) Desrosières A. La politique des grands nombres. Histoire de la raison statistique. $2^{\text {nd }}$. Edition. Paris: La Découverte, 2000.

(17) Álvarez JM, Colina F, Esteban R. Presentación de esta selección del Manual de Semiología de las Enfermedades Mentales de Enrico Morselli. En: Morselli E. Manual de semiología de las enfermedades mentales (selección). Madrid: Alienistas del Pisuerga, 2012; p. XI-XVII.

(18) Lantéri-Laura G. Introduction générale. Evol Psych 2005; 70: 219-247.

(19) Huertas R. El siglo de la clínica: para una teoría de la práctica psiquiátrica. Madrid: Frenia, 2005.

(20) Desviat M. Síntoma, signo e imaginario social. Rev Asoc Esp Neuropsiqu 2010; 30: 125-133.

(21) Rullier P. Faculté. En: Dictionnaire des sciences médicales-par une société des médecins et de chirurgiens. Vol. 14. Paris: C.L.F. Panckoucke, 1815; p. 389-420. 